\title{
Focal Adhesion Kinase Is Required, But Not Sufficient, for the Induction of Long-Term Potentiation in Dentate Gyrus Neurons In Vivo
}

\author{
Ying C. Yang, ${ }^{1}$ Yun L. Ma, ${ }^{2}$ Shau K. Chen, ${ }^{2}$ Cheng W. Wang, ${ }^{3}$ and Eminy H. Y. Lee ${ }^{2}$ \\ ${ }^{1}$ Graduate Institute of Life Sciences, National Defense Medical Center, Taipei 114, Taiwan, ${ }^{2}$ Institute of Biomedical Sciences, Academia Sinica, Taipei 115, \\ Taiwan, and ${ }^{3}$ Department of Zoology, National Taiwan University, Taipei 106, Taiwan, Republic of China
}

Tyrosine kinase phosphorylation plays an important role in the induction of long-term potentiation (LTP). Focal adhesion kinase (FAK) is a $125 \mathrm{kDa}$ nonreceptor tyrosine kinase that shows decreased phosphorylation in fyn mutant mice, and Fyn plays a critical role in LTP induction. By examining the role of FAK involved in LTP induction in dentate gyrus in vivo with medial perforant path stimulation, we found that both FAK and mitogen-activated protein kinase (MAPK)/extracellular signal-regulated kinase (ERK) phosphorylation were increased significantly 5 and 10 min after LTP induction, whereas cAMP-responsive element binding protein (CREB) phosphorylation was increased 40 min later. Transfection of the dominant-negative FAK mutant construct HA-FAK(Y397F) impaired LTP, whereas transfection of the constitutively activated form HA-FAK $(\Delta 1-100)$ reduced the threshold for LTP induction. Transfection of HAFAK $(\Delta 1-100)$ by itself did not induce long-lasting potentiation. Further, transfection of the HA-FAK(Y397F) construct decreased FAK, MAPK/ERK, and CREB phosphorylation, and the inhibition of MAPK/ERK decreased CREB phosphorylation. Moreover, blockade of NMDA receptor (NMDAR) did not decrease FAK, MAPK/ERK, and CREB phosphorylation although LTP induction was blunted by NMDAR antagonist. These biochemical changes were not associated with low-frequency stimulation either. Immunoprecipitation results revealed that tyrosine phosphorylation of NR2A and NR2B as well as the association of phosphorylated FAK with NR2A and NR2B was increased with LTP induction. These results together suggest that FAK is required, but not sufficient, for the induction of LTP in a NMDAR-independent manner and that MAPK/ERK and CREB are the downstream events of FAK activation. Further, FAK may interact with NR2A and NR2B to modulate LTP induction.

Key words: focal adhesion kinase; long-term potentiation; mitogen-activated protein kinase/extracellular signal-regulated kinase; cAMPresponsive element binding protein; field EPSP; NMDA receptor; metabotropic glutamate receptor; phosphorylation

\section{Introduction}

Accumulative evidence has indicated that protein tyrosine phosphorylation is necessary for the induction of long-term potentiation (LTP) (Soderling and Derkach, 2000). For example, application of protein tyrosine kinase inhibitor has been shown to block LTP induction (O'Dell et al., 1991), whereas activation of tyrosine receptor kinase contributes to the expression of LTP (Maguire et al., 1999). The NMDA receptor (NMDAR) is known to play an essential role in LTP induction (Collingridge and Bliss, 1995). Evidence indicates that NMDARs are regulated by tyrosine phosphorylation (Lau and Huganir, 1995) and that LTP in dentate gyrus is correlated specifically with tyrosine phosphorylation of the NMDAR subunit 2B (NR2B) (Rosenblum et al., 1997). These results suggest that signaling via tyrosine kinase plays an important role in the synaptic plasticity of hippocampal neurons.

Tyrosine kinases can be divided into two categories. One is the membrane-spanning receptor tyrosine kinase that transduces signals from growth factors and neurotrophic factors. The other

Received Aug. 13, 2002; revised Feb. 19, 2003; accepted Feb. 24, 2003. This work was supported by a research fund from the Institute of Biomedical Sciences, Academia Sinica, Taiwan, the Republic of China.

Correspondence should be addressed to Dr. Eminy H. Y. Lee, Institute of Biomedical Sciences, Academia Sinica, Taipei 115, Taiwan, Republic of China. E-mail: eminy@ibms.sinica.edu.tw.

Copyright $\odot 2003$ Society for Neuroscience $\quad$ 0270-6474/03/234072-09\$15.00/0 is the nonreceptor tyrosine kinase associated with the cytoplasmic domain of the plasma membrane (Koch et al., 1991). Study shows that deletion of the gene encoding the Fyn nonreceptor tyrosine kinase impairs LTP in fyn mutant mice (Grant et al., 1992). This impairment in LTP is rescued by introducing the fyn gene even though the morphological abnormalities are still present (Kojima et al., 1997). On the other hand, overexpression of the constitutively activated mutant of $f y n$ reduces the threshold for LTP induction and enhances synaptic transmission (Lu et al., 1998). In addition, transgenic mice express a constitutively activated form of fyn that enhances tyrosine phosphorylation of NR2B (Kojima et al., 1998). These results suggest that the nonreceptor tyrosine kinase Fyn is important for the induction of LTP and for the phosphorylation of NMDARs.

There are nine proteins that show decreased phosphorylation in fyn mutant mice; one of these proteins is focal adhesion kinase (FAK) (Grant et al., 1995). FAK is a $125 \mathrm{kDa}$ nonreceptor tyrosine kinase that is expressed maximally in the CNS at the end of embryonic development. It also is found in the adult brain at levels higher than those in other tissues, especially in the cerebral cortex and hippocampus (Burgaya et al., 1995). In primary cultures FAK is found to localize in focal adhesions, whereas in mature neurons FAK is distributed in the cytoplasm and growth cones (Burgaya et al., 1995). These observations suggest that FAK may be impli- 
cated in neural plasticity in the adult brain. Indeed, an increase in Fyn phosphorylation and the association of Fyn with FAK have been reported in CA1 neurons after tetanic stimulation (Lauri et al., 2000). Tyrosine phosphorylation of FAK also is increased markedly with glutamate stimulation in hippocampal slices ( $\mathrm{Si}$ ciliano et al., 1996). Although the above results suggest a possible association between FAK and LTP, a direct relationship between FAK and LTP has not been demonstrated. Whether FAK is associated with the NMDAR also is not known. The present study was aimed to examine whether FAK plays an important role in LTP induction and whether FAK is associated with NMDAR phosphorylation at LTP induction.

\section{Materials and Methods}

Animals. Adult male Sprague Dawley rats, weighing 250-350 gm, bred in the Institute of Biomedical Sciences, Academia Sinica in Taiwan, were housed five per cage in a temperature-regulated room $\left(23 \pm 2{ }^{\circ} \mathrm{C}\right)$ and maintained on a $12 \mathrm{hr}$ light/dark cycle (lights on at 6:30 A.M.) with food and water continuously available. All procedures were adopted according to the Guidelines of Animal Use and Care of National Institutes of Health.

LTP recording and drug administration. The procedure used for LTP recording in dentate gyrus neurons was according to that of Wang et al. (1998) with minor modifications. Rats were anesthetized with urethane $(1.4 \mathrm{gm} / \mathrm{kg}$, i.p. $)$ and placed on a stereotaxic instrument. Platinum concentric bipolar-stimulating electrodes were positioned unilaterally into the dorsomedial perforant pathway at $8.3 \mathrm{~mm}$ posterior to bregma, 4.4 $\mathrm{mm}$ lateral to the midline, and $2.7 \mathrm{~mm}$ below the skull surface. The recording electrodes were positioned $3.5 \mathrm{~mm}$ posterior to bregma and 2.0 $\mathrm{mm}$ from the midline. Electrodes were positioned to maximize hilar field potentials evoked by medial perforant path stimulation. Stimulation was $50 \mu \mathrm{sec}$ in duration pulses delivered at a frequency of $0.033 \mathrm{~Hz}$. An input-output curve was obtained over the range of $50-400 \mu \mathrm{A}$. Stimulus intensities ranged from 125 to $250 \mu \mathrm{A}$ and produced an average field EPSP (fEPSP) amplitude of 6-10 mV. For induction of LTP four sets of tetanic stimulation were delivered at $10 \mathrm{~min}$ intervals after a $30 \mathrm{~min}$ baseline recording. Each set consisted of five trains of 10 pulses at $400 \mathrm{~Hz}$ with $1 \mathrm{sec}$ between trains. The pulse widths in the four sets of trains were $50,100,150$, and $200 \mu \mathrm{sec}$, respectively. The subthreshold stimulation consisted of only two sets of tetanic stimulation with pulse widths of 50 and $100 \mu \mathrm{sec}$, respectively. In a separate series of experiments the effect of low-frequency stimulation (LFS) was assessed. For LFS each set consisted of 50 pulses at $2 \mathrm{sec}$ intervals. The pulse widths in the four sets of trains were 50,100,150, and $200 \mu \mathrm{sec}$, respectively. Control rats received baseline stimulation only. LTP was measured as the percentage of baseline in the initial slope of fEPSP at $1.52 \mathrm{msec}$.

A $100 \mathrm{~mm}$ stock solution of D,L-2-amino-5-phosphopentonate (D,LAPV, Tocris, Bristol, UK) was made up in equimolar $\mathrm{NaOH}$ and was diluted to $200 \mu \mathrm{M}$ with artificial CSF (ACSF). A $1 \mathrm{~m}$ stock solution of $(R, S)$ - $\alpha$-methyl-4-carboxylphenylglycine (MCPG) was prepared in equimolar $\mathrm{NaOH}$ and was diluted to $200 \mathrm{~mm}$ with ACSF. MEK inhibitor (PD98059, Calbiochem, San Diego, CA) was dissolved in 100\% DMSO for a $20 \mathrm{~mm}$ stock solution, and a final concentration of $75 \mu \mathrm{M}$ PD98059 ( $0.375 \%$ DMSO) was diluted with ACSF. Drugs were infused ( $0.8 \mu \mathrm{l}, 0.25$ $\mu \mathrm{l} / \mathrm{min}$ ) to the recording site, and LTP was recorded 10 min later.

Synaptosome preparation and immunoprecipitation. The dentate gyrus was homogenized in PBS, $\mathrm{pH} 7.4$, containing $0.32 \mathrm{~m}$ sucrose, using a Teflon glass homogenizer. The homogenate was centrifuged at $1000 \times g$ for $10 \mathrm{~min}$ to discard the nuclei pellet. Then the supernatant was spun at $12,000 \times g$ for $30 \mathrm{~min}$, and the resulting pellet (P2) containing synaptosomes was resuspended in $50 \mathrm{~mm}$ Tris- $\mathrm{HCl}, \mathrm{pH}$ 8.0, $150 \mathrm{~mm} \mathrm{NaCl}$ buffer containing (in mM) 2 EDTA, $1 \mathrm{Na}_{3} \mathrm{VO}_{4}, 10 \mathrm{NaF}$, and 1 PMSF plus (in $\mu \mathrm{g} / \mathrm{ml}) 20$ pepstatin A, 20 leupeptin, and 20 aprotinin, followed by incubation for $30 \mathrm{~min}$. For immunoprecipitation, $100 \mu \mathrm{g}$ of crude synaptosomes was precleared with normal goat anti-rabbit IgG and protein G-Sepharose (Amersham Biosciences, Arlington Heights, IL) twice. Once precleared, anti-FAK $\left[\mathrm{pY}^{397}\right]$ (7 $\mu \mathrm{g}$; BioSource, Camarillo, CA), NR2A, or NR2B ( $5 \mu \mathrm{g}$; Chemicon, Temecula, CA) antibodies were added to the supernatant for at least $15 \mathrm{hr}$ at $4^{\circ} \mathrm{C}$. Then $120 \mu \mathrm{l}$ of $50 \%$ protein $\mathrm{G}$-Sepharose was added for $2 \mathrm{hr}$ at $4^{\circ} \mathrm{C}$. The immunoprecipitates were isolated by centrifugation, washed three times with binding buffer, and washed once with PBS. The beads were boiled in $2.5 \times$ Laemmli sample buffer (with DTT) for $10 \mathrm{~min}$.

Immunoblotting. The dentate gyrus was sonicated in lysis buffer containing (in mM) 50 Tris- $\mathrm{HCl}, \mathrm{pH} 8.0,150 \mathrm{NaCl}, 2$ EDTA, $1 \mathrm{Na}_{3} \mathrm{VO}_{4}, 10$ $\mathrm{NaF}$, and 1 PMSF plus $1 \% \mathrm{NP}-40$ and (in $\mu \mathrm{g} / \mathrm{ml}$ ) 20 pepstatin A, 20 leupeptin, and 20 aprotinin. The lysate was centrifuged at $14,000 \times g$ for $10 \mathrm{~min}$ at $4^{\circ} \mathrm{C}$ to get the crude cytosolic fraction. For cAMP-responsive element binding protein (CREB) determination the dentate gyrus was homogenized in buffer containing $0.32 \mathrm{M}$ sucrose and (in mM) 20 Tris$\mathrm{HCl}, \mathrm{pH}$ 7.4, 1 EDTA, 1 EGTA, 1 PMSF, $1 \mathrm{Na}_{3} \mathrm{VO}_{4}$, and $10 \mathrm{NaF}$ plus (in $\mu \mathrm{g} / \mathrm{ml}) 20$ pepstatin A, 20 leupeptin, and 20 aprotinin. The homogenate was centrifuged at $900 \times g$ for $10 \mathrm{~min}$, and the pellet was collected as the nuclear fraction.

Equal amounts of sample extract $(25 \mu \mathrm{g})$ and $15 \mu \mathrm{g}$ of synaptosome extract or immune complex were subjected to SDS-PAGE and transferred to the polyvinylidene difluoride membrane (Millipore, Bedford, MA). The membrane was blocked with $2 \%$ BSA and then incubated with anti-phosphotyrosine (mAb 4G10), phospho-extracellular signalregulated kinase (ERK) $1 / 2$, phospho-CREB, ERK1/2 or CREB, phosphatidylinositol 3-kinase (PI3-kinase; Upstate Biotechnology, Lake Placid, NY), NR1, NR2A, NR2B (Chemicon), and FAK $\left[\mathrm{pY}^{397}\right]$ (BioSource) antibodies. After being washed, the blot was incubated with HRPconjugated secondary antibody and was visualized via chemiluminescence (Amersham Biosciences). For reprobing, the blot was stripped in $0.2 \mathrm{M}$ glycine, $\mathrm{pH} 2.5$, and $0.05 \%$ Tween 20 at $80^{\circ} \mathrm{C}$ for $30 \mathrm{~min}$ and then rinsed twice with $0.09 \mathrm{M}$ boric acid, $\mathrm{pH} 7.4,0.9 \% \mathrm{NaCl}$, and $0.05 \%$ Tween 20. The membrane was reblocked and incubated with the monoclonal antibody against FAK (clone 2A7; Upstate Biotechnology). Biotinylated rabbit anti-mouse IgG antibody (DAKO, Glostrup, Denmark) was used as the secondary antibody. Finally, the membrane was incubated with HRP-conjugated avidin-biotin complex (DAKO). The protein bands were quantified by using a computing densitometer with ImageQuant software (Molecular Dynamics, Sunnyvale, CA).

Site-directed mutagenesis and plasmid DNA construction. The fulllength mouse FAK cDNA containing triple hemagglutinin (HA) epitope tag at the $\mathrm{C}$ terminus and cloned in KpnI-XbaI-digested pcDNA3 CMV promoter-driven expression vector [HA-FAK(WT); Invitrogen, San Diego, CA] was generated. Site-directed mutagenesis was used to produce the HA-FAK(Y397F) construct, the kinase-dead and the dominantnegative mutant construct of FAK in which the Tyr-397 phosphoacceptor site was changed to the phenylalanine codon by the PCR method. In addition, site-directed mutagenesis was used to produce the HA$\mathrm{FAK}(\Delta 1-100)$ construct, the constitutively activated form of FAK, also by the PCR method according to that described by Schlaepfer and Hunter (1996).

Preparation of DNA/PEI complexes and intrahippocampal gene transfection. Plasmid DNA was diluted in $5 \%$ glucose, aliquoted at concentrations of $5 \mu \mathrm{g} / \mu \mathrm{l}$, and stored at $-20^{\circ} \mathrm{C}$. Polyethylenimine (PEI) of $25 \mathrm{kDa}$ (Aldrich, Milwaukee, WI) was used as the transfection agent. Immediately before infusion, $0.1 \mathrm{M}$ PEI was added to reach a ratio of PEI nitrogen per DNA phosphate equal to 10 . The mixture was vortexed for $30 \mathrm{sec}$ and allowed to equilibrate for $15 \mathrm{~min}$ at room temperature (RT) before stereotaxic infusion (Abdallah et al., 1996).

Rats were anesthetized with sodium pentobarbital (50 mg/kg, i.p.) and received unilateral intradentate gyrus infusions. Infusions were made at $3.5 \mathrm{~mm}$ posterior to bregma, $2.0 \mathrm{~mm}$ from the midline, and $3.5 \mathrm{~mm}$ below the skull surface. The plasmid DNA/PEI $(1 \mu \mathrm{l})$ was injected at a rate of $0.4 \mu \mathrm{l} / \mathrm{min}$. Control rats received the nonrecombinant pcDNA3 and PEI mixture in the same conditions. LTP recording ipsilateral to the transfection side started $48 \mathrm{hr}$ later.

Immunohistochemistry. At $2 \mathrm{~d}$ after transfection the rats were anesthetized with pentobarbital $(100 \mathrm{mg} / \mathrm{kg}$, i.p.) and perfused with ice-cold PBS buffer (100 mM, pH 7.4), followed by $4 \%$ paraformaldehyde. Brains were removed and postfixed in $20 \%$ sucrose $/ 4 \%$ paraformaldehyde solution for $20-48 \mathrm{hr}$. After postfixation, brains were frozen, cut into $20 \mu \mathrm{m}$ sections on a cryostat, and mounted on poly-L-lysine-coated slides. Sec- 
A

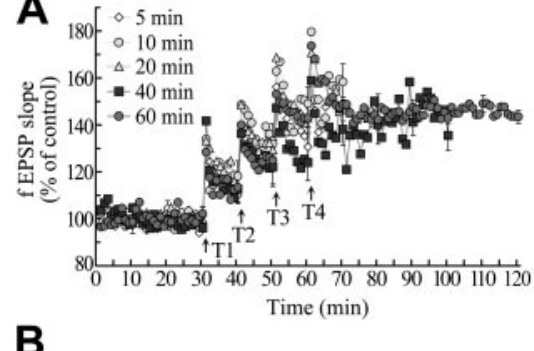

B
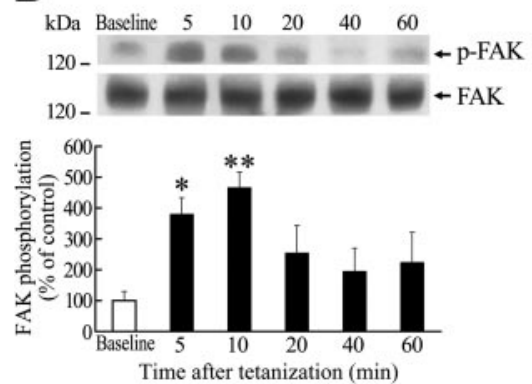

C
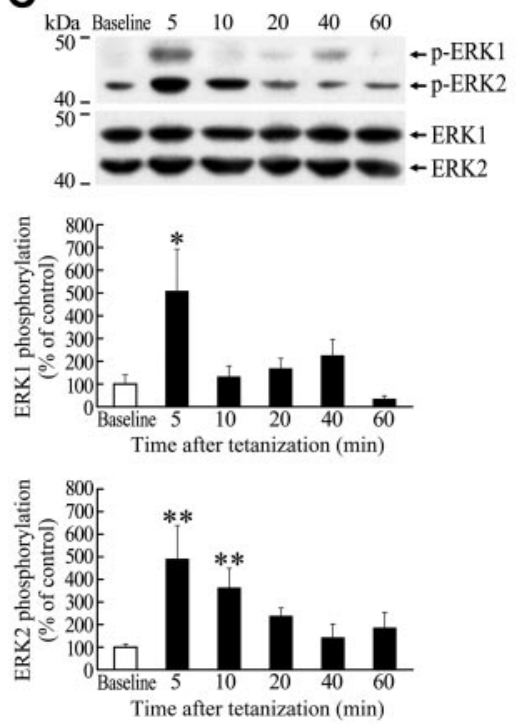

D
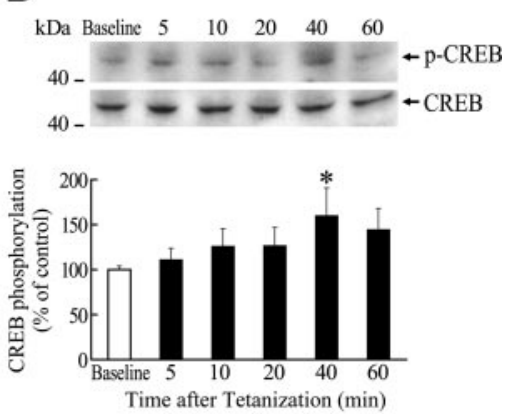

Figure 1. LTP induction increases FAK, ERK, and CREB phosphorylation. $A$, High-frequency stimulation of the medial perforant pathway consistently induces LTP at different time points. T, Train of stimulation. $B$, Western blots showing that FAK phosphorylation was increased markedly 5 and 10 min after LTP induction; $n=6$ for each group. $C$, p-ERK1 was increased markedly 5 min after LTP induction, and p-ERK2 was increased 5 and 10 min after LTP induction. D, p-CREB was increased significantly 40 min after LTP induction; $n=8$ for each group in $C, D$. Data are expressed as mean \pm SEM; ${ }^{*} p<0.05$ and ${ }^{* *} p<0.01$, one-way ANOVA and Dunnett's $t$ test when compared with the control group.

tions were permeabilized in prechilled $95 \% \mathrm{ETOH} / 5 \% \mathrm{CH}_{3} \mathrm{COOH}$ for 5 min, followed by preincubation with $4 \%$ normal goat serum, $3 \%$ BSA, and $0.2 \%$ Triton X-100 for $1 \mathrm{hr}$ at RT. Then the sections were incubated with mouse 12CA5 monoclonal anti-HA antibody ( Boehringer Mannheim, Indianapolis, IN) at $4^{\circ} \mathrm{C}$ overnight. Next the FITC-conjugated secondary antibody (Sigma, St. Louis, MO) was used for incubation for 1 hr at RT. Digital photomicrographs were taken with an Olympus digital C-3030 camera mounted on a Zeiss (Oberkochen, Germany) microscope. Digital images were adjusted with the Adobe Photoshop software (Mountain View, CA).

\section{Results}

LTP induction induces FAK, MAPK/ERK, and CREB activation

Because the fyn mutant mice show impaired LTP (Grant et al., 1992) and decreased FAK phosphorylation (Grant et al., 1995) and because of the association of Fyn with FAK with tetanic stimulation (Lauri et al., 2000), we examined the relationship between LTP induction and FAK activation in this experiment. The dentate gyrus tissue from the stimulation side was punched out at 5 , $10,20,40$, and $60 \mathrm{~min}$ after tetanic stimulation in separate groups of animals. Figure $1 A$ shows that tetanic stimulation consistently increased the slope of fEPSP in all groups of rats (ranging from 128 to $180 \%$ of baseline). Figure $1 B$ illustrates the representative gel pattern of FAK and phosphorylated FAK bands $(n=6$ for each group). Densitometry analysis revealed that there was an overall significant effect of tetanic stimulation on FAK phosphorylation $\left(F_{(5,30)}=3.80 ; p<0.01\right)$. Further analyses revealed that FAK phosphorylation was increased transiently but significantly at 5 and 10 min after LTP induction (3.8-fold increase, $t D=2.93$, $p<0.05 ; 4.6$-fold increase, $t D=3.82, p<0.01)$ and gradually declined thereafter. Meanwhile, the FAK protein level remained relatively the same at all time points that were examined (Fig. $1 B)$.

Previous studies have shown that LTP-inducing stimuli produce mitogen-activated protein kinase (MAPK) activation (English and Sweatt, 1996) and that inhibition of MAPK blocks the induction of LTP in hippocampal slices (English and Sweatt,
1996, 1997). Recent studies have shown further that MAPK/ERK activation contributes to LTP and other forms of synaptic plasticity in hippocampal neurons (Coogan et al., 1999; Davis et al., 2000). However, the time course relationship between LTP induction and MAPK/ERK activation has not been characterized yet. We examined this relationship in the present experiment. Both forms of MAPK/ERK, p44/ERK1 and p42/ERK2, were examined. Figure $1 C$ illustrates the representative gel pattern of ERK1 and ERK2 bands as well as phosphorylated ERK1 and ERK2 bands ( $n=8$ for each group). Densitometry analysis revealed that ERK1 phosphorylation was increased transiently but significantly $5 \mathrm{~min}$ after tetanic stimulation (fivefold increase, $t D=3.30, p<0.05$; Fig. $1 C$ ). There was an overall significant effect of tetanic stimulation on ERK2 phosphorylation $\left(F_{(5,42)}=\right.$ $4.75 ; p<0.01)$. Further analyses revealed that ERK2 phosphorylation was increased markedly at 5 and $10 \mathrm{~min}$ after tetanization (5.1-fold increase, $t D=4.17, p<0.01$ and 3.6-fold increase, $t D=$ $2.24, p<0.01$; Fig. $1 C$ ). This change in time course parallels with that of FAK phosphorylation (Fig. $1 B$ ). Meanwhile, the protein levels of ERK1 and ERK2 remained unchanged at all time points that were examined (Fig. $1 C$ ).

Inhibition of MAPK/ERK has been shown to block LTPinduced CREB activation (Davis et al., 2000), and CREB phosphorylation is suggested to regulate short- to long-term synaptic changes (Abel et al., 1998). We similarly have examined the time course relationship between LTP induction and CREB activation. Results revealed that there was not an overall significant effect of tetanic stimulation on CREB activation $\left(F_{(5,30)}=0.41 ; p>0.05\right)$; however, CREB phosphorylation was increased markedly at 40 min, but not other time points, after LTP induction (1.6-fold increase, $t D=2.04, p<0.05$; Fig. $1 D$ ). The protein levels of CREB were similar in all groups that were examined (Fig. 1D).

\section{Dominant-negative mutant of FAK attenuates} tetanization-induced LTP

Results from the above experiment have demonstrated a timedependent change of FAK phosphorylation with LTP induction. 

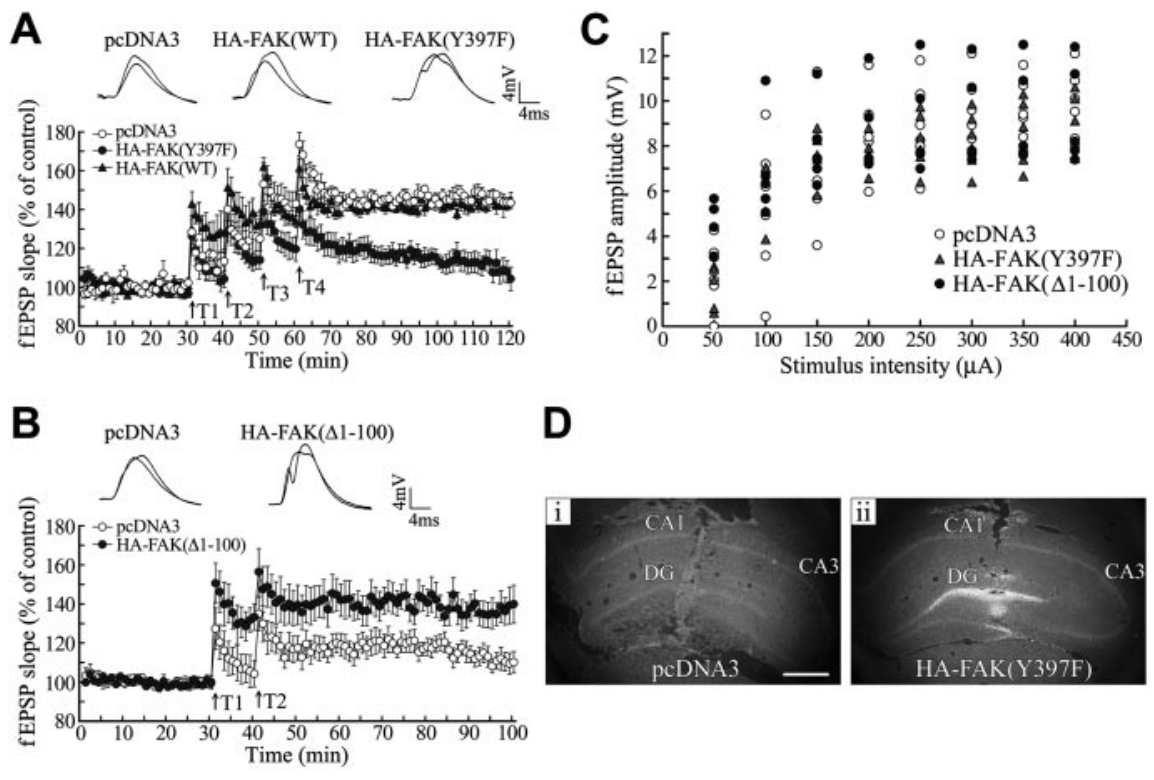

Figure 2. FAK alters the threshold of LTP induction. A, In animals transfected with the HA-FAK(Y397F) construct $48 \mathrm{hr}$ previously, the slope of fEPSP was decreased significantly when compared with the pCDNA3 transfection group and the HA-FAK(WT) transfection group (both $p<0.01$ ). Insets, Representative fEPSP at baseline and 60 min after tetanic stimulation from control ( $p$ cDNA3 vector), HA-FAK(WT)-, and HA-FAK(Y397F)-transfected rats; $n=5-10$ for each group. $B$, In animals transfected with the HA-FAK $(\Delta 1-100)$ construct, LTP induced by subthreshold stimulation was potentiated ( $140 \%$ of baseline), and it was significantly different from the vector-only transfection group ( $117 \%$ of baseline); $p<0.05$. Insets, Representative fEPSP from control and HA-FAK $(\Delta 1-100)$-transfected rats; $n=5-6$ for each group. Statistics were evaluated by one-way ANOVA $(A)$ and Student's $t$ test $(B)$, respectively. $C$, Comparison of postsynaptic responses under different stimulation intensities among pcDNA3-, HAFAK(Y397F)-, and HA-FAK( $\Delta 1-100)$-transfected rats; $n=5-6$ for each group. No significant difference was found among these treatments at any stimulus intensity that was examined (all $p>0.05$ ). Data represent individual animals. D, Immunohistochemistry showing $\mathrm{HA}$ staining in the dentate gyrus. $\mathrm{Di}$, No specific HA staining was observed in the dentate gyrus in pCDNA3 vectortransfected animals. Dii, HA epitope-positive cells were detected with FITC-conjugated secondary antibody in dentate gyrus transfected with the HA-FAK(Y397F) construct; $n=5$ for each group. Scale bar, $500 \mu \mathrm{m}$.

If FAK plays an important role in the induction of LTP, blockade of FAK activation should impair LTP. Because FAK deficiency is lethal at developmental stages (Furuta et al., 1995), we have transfected the dominant-negative mutant of FAK, HA-FAK(Y397F), to the dentate gyrus and examined its effect on LTP induction. The nonviral PEI gene transfer technique was used because of its lack of toxicity and its efficiency in transferring plasmid DNA into the adult brain (Boletta et al., 1995; Abdallah et al., 1996). A complex consisting of $2 \mu \mathrm{g}$ of plasmid DNA and $25 \mathrm{kDa}$ PEI at a charge ratio of 10 equivalents was infused into the dentate gyrus $(0.4 \mu \mathrm{l} / \mathrm{min})$, and LTP was recorded $2 \mathrm{~d}$ later. At the end of recording the animals were subjected to immunohistochemistry staining of HA by using FITC-conjugated secondary antibody. As shown in Figure $2 A$, transfections of pcDNA3 vector $(n=5)$ and the HA-FAK(WT) construct $(n=6)$ both produced a consistent increase in the slope of fEPSP (61-120 $\mathrm{min}$ ) (147 and $143 \%$ of baseline, $t D=63.8$ and $42.3 ; p<0.01)$. However, transfection of the HA-FAK(Y397F) construct $(n=10)$ significantly reduced the slope of fEPSP (61-120 min) when compared with the pcDNA3 vector group ( 117 vs $147 \%$ of baseline, $t D=5.15 ; p<0.01$ ).

\section{Constitutively activated FAK construct reduces the threshold for LTP induction}

The above results demonstrate that FAK plays an important role in the induction of LTP. In the present experiment we further examined whether FAK affects the threshold for LTP induction. Two sets, instead of four sets, of tetanic stimulation were delivered to animals to elicit reduced magnitude of LTP. As shown in Figure $2 B$, this subthreshold stimulation induced a lesser degree of postsynaptic excitation (41-100 min; $117 \%$ of baseline; $n=6$ ). However, transfection of the constitutively activated form of FAK, HA-FAK $(\Delta 1-100)$, significantly increased the slope of fEPSP (41-100 $\mathrm{min})$ [ $140 \%$ of baseline, $n=5\left(t_{(1,9)}=2.42 ; p<\right.$ $0.05)$ when compared with the pcDNA3 vector group]. Further analysis revealed that this effect was significant as early as the first train (T1) stimulation $\left(t_{(1,9)}=\right.$ 1.96; $p<0.05)$. Moreover, a population spike of dentate gyrus neurons was observed more frequently in $\operatorname{HA}-\mathrm{FAK}(\Delta 1-$ $100)$-transfected animals with tetanic stimulation (insets of Fig. $2 B$ ).

We further analyzed whether FAK alters basal synaptic transmission of dentate gyrus neurons. By examining the relationship between stimulation intensity and postsynaptic response, we found that neither HA-FAK(Y397F) nor HA-FAK $(\Delta 1-$ 100) transfection significantly altered the input-output relationship across a wide range of stimulation intensities that were examined $\left(F_{(2,14)}=0.26 ; p>0.05\right.$; Fig. 2C).

To confirm the efficiency of FAK plasmid DNA transfection, we have conducted an immunohistochemistry experiment that used FITC-conjugated secondary antibody to detect HA epitope-positive cells. Results revealed that no fluorescence labeling was observed in non-HA vectortransfected animals. However, fluorescence labeling of dentate gyrus neurons was observed clearly in HA-FAK DNA-transfected animals (Fig. 2D).

\section{LTP-induced FAK phosphorylation is NMDAR- and mGluR-independent}

The above results demonstrated that LTP induction induces FAK phosphorylation. Because both the NMDAR and mGluR are suggested to be involved in LTP induction (Bashir et al., 1993), in the present experiment we examined whether LTP-induced FAK phosphorylation is dependent on NMDAR and mGluR activation. D,L-APV, a competitive antagonist of NMDAR, was infused to the dentate gyrus $10 \mathrm{~min}$ before LTP recording. Results revealed that D,L-APV by itself $(200 \mu \mathrm{M})$ did not affect basal transmission, but it markedly inhibited LTP induction (61-65 min) [ 125 vs $158 \%$ of baseline, $n=7$ and $10(t D=2.6 ; p<0.05)$ when compared with the ACSF group]. However, the broad-spectrum mGluR antagonist MCPG (200 mM) given $10 \mathrm{~min}$ before did not inhibit LTP induction (61-65 min) [ 163 vs $158 \%$ of baseline, $n=$ 7 and $10(t D=0.35 ; p>0.05)$ when compared with the ACSF group]. The dentate gyrus was dissected out $5 \mathrm{~min}$ after LTP induction and was subjected to Western blot analysis. Results showed that neither D,L-APV nor MCPG significantly inhibited LTP-induced FAK phosphorylation [D,L-APV vs ACSF $\left(q_{(2,24)}=\right.$ $69 ; p>0.05)$; MCPG vs ACSF, $\left(q_{(3,24)}=79.6 ; p>0.05\right)$, by Newman-Keuls method] (Fig. $3 A$ ). To clarify the specific relationship between FAK phosphorylation and LTP induction, we have included the LFS group as a control. Results revealed that LFS did not affect fEPSP slope and FAK phosphorylation $(t D=$ 1.14 and 0.04 ; both $p>0.05$; Fig. $3 A$ ). 

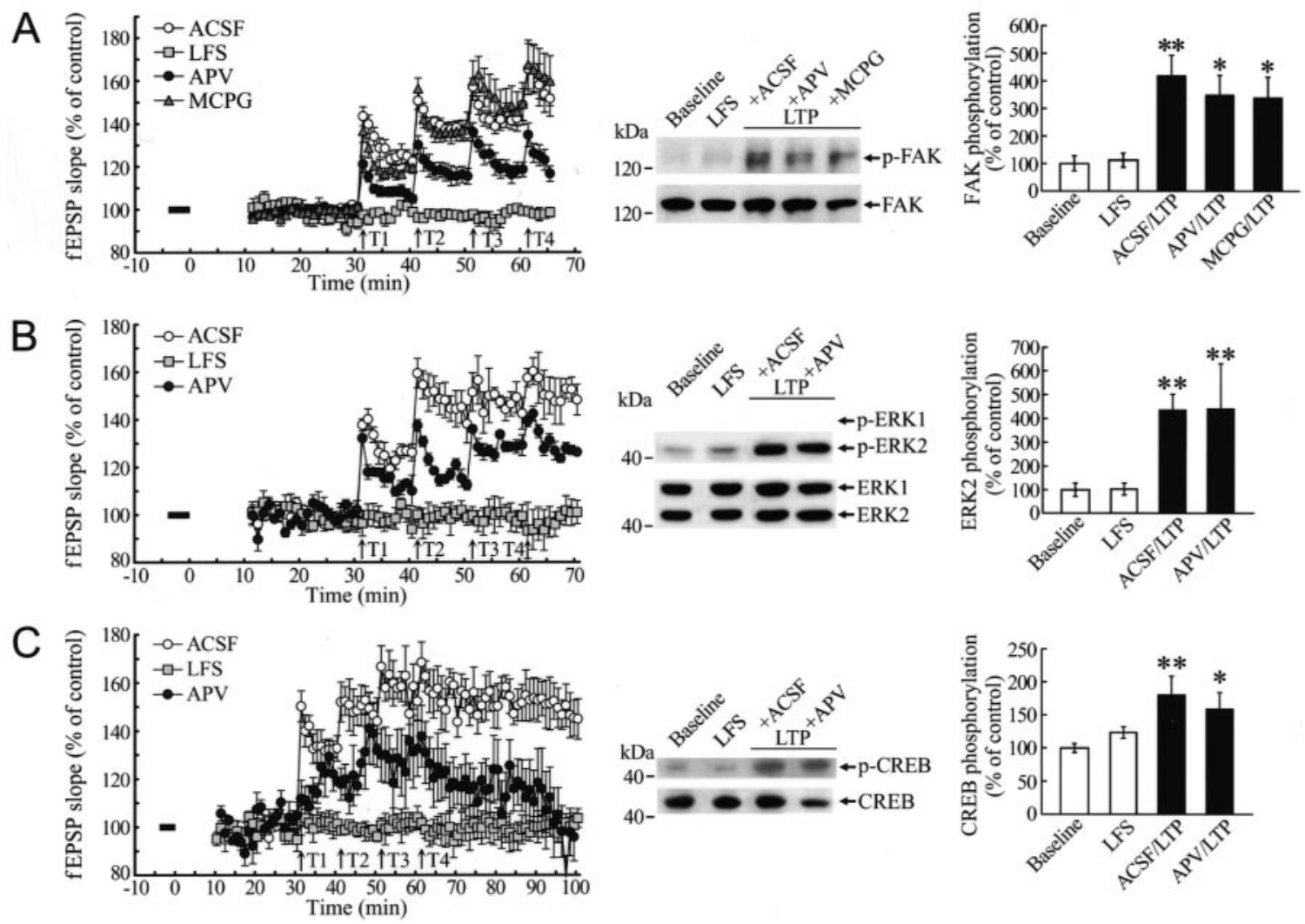

Figure 3. LTP-induced, but not LFS-induced, FAK phosphorylation is NMDAR- and mGluR-independent, and ERK and CREB are downstream events of FAK activation. $A$, Injection of $D, L-A P V ~(200$ $\mu \mathrm{M}, 0.8 \mu \mathrm{l})$ to dentate gyrus 10 min before LTP recording (horizontal black bar) markedly decreased the slope of fEPSP $(p<0.05)$, whereas injection of MCPG ( $200 \mathrm{mM})$ failed to inhibit LTP induction $(p>0.05)$. FAK phosphorylation was not altered markedly in $D, L-A P V$ - and MCPG-injected rats when compared with the ACSF group (both $p>0.05)$. LFS did not alter the slope of fEPSP and FAK phosphorylation; $n=7$ for each group. $B, \mathrm{D}, \mathrm{L}-\mathrm{APV}$ injection significantly blocked LTP induction $(p<0.01$ ), but it did not block LTP-induced ERK2 phosphorylation ( $p<0.01$ when compared with the control group); $n=4$ for each group. C, D,L-APV injection markedly impaired LTP induction $(p<0.01)$, but it only partially inhibited LTP-induced CREB phosphorylation $(p<0.05) ; n=4-5$ for each group. Data are expressed as in Figure 1. ${ }^{*} p<0.05 ;{ }^{* *} p<0.01$. Statistics were calculated by one-way ANOVA, followed by Dunnett's $t$ test or Newman-Keuls method.

The above results revealed that LTP induction, but not LFS, induces FAK phosphorylation. To examine further whether MAPK/ERK and CREB phosphorylation is a specific event with LTP induction, we have adopted the D,L-APV manipulation and LFS as a control in a separate group of rats ( $n=4$ for each group). Results from Figure $3 B$ revealed that high-frequency stimulation produced a consistent and marked increase in the slope of fEPSP $(t D=15.74 ; p<0.01)$, which was blocked by previous D-APV treatment. LTP induction also induced a significant increase in ERK2 phosphorylation $(t D=2.98 ; p<0.01)$, but this effect was not blocked by D,L-APV pretreatment $(t D=3.02 ; p<0.01$ when comparing the D,L-APV + LTP group with the control group). On the other hand, LFS did not affect fEPSP slope and ERK2 phosphorylation markedly $(t D=0.62$ and 0.05 ; both $p>0.05)$.

We next examined the causal relationship between LTP induction and CREB phosphorylation in a different batch of animals by using the same strategy ( $n=4-5$ for each group). Results from Figure $3 C$ revealed that high-frequency stimulation produced a consistent increase in fEPSP slope $(t D=13.73 ; p<0.01)$, which was blocked by D,L-APV pretreatment. LTP induction also induced a marked increase in CREB phosphorylation $(t D=3.31$; $p<0.01$ ), but this effect was inhibited only partially by previous D,L-APV treatment $(t D=2.06$; $p<0.05$ when comparing the D,L-APV+LTP group with the control group). Consistent with the above results, LFS did not alter fEPSP slope and CREB phosphorylation markedly $(t D=1.93$ and 0.63 ; both $p>0.05)$.

\section{MAPK/ERK and CREB are downstream molecules of FAK activation with LTP induction}

Although the above results demonstrated that LTP induction induces both FAK and MAPK/ERK activations and the time course of these activations parallels each other, it is not known whether MAPK/ERK is a downstream event of FAK activation with LTP induction. In the present experiment LTP was recorded similarly $2 \mathrm{~d}$ after HA-FAK(Y397F) transfection to the dentate gyrus. Then the dentate gyrus was dissected out $10 \mathrm{~min}$ after LTP induction for FAK and ERK determinations. As shown in Figure 4A, HAFAK(Y397F) transfection produced a consistent and significant decrease of fEPSP slope when compared with the pcDNA3 vector group (61-70 $\mathrm{min}$ ) [ $122 \mathrm{vs} 157 \%$ of baseline, $n=7$ and $5\left(t_{(1,10)}\right.$ $=3.7 ; p<0.01)]$. Further densitometry analysis revealed that there was a significant decrease in FAK phosphorylation $\left(t_{(1,10)}=\right.$ $2.89 ; p<0.01$; Fig. $4 A$, middle panel) and ERK2 phosphorylation $\left(t_{(1,10)}=2.92 ; p<0.01\right.$; Fig. $4 \mathrm{~A}$, bottom panel $)$ in HAFAK(Y397F)-transfected animals. The protein levels for FAK and ERK2 remained similar in these two groups of animals. We then investigated whether CREB is a downstream event of FAK activation with LTP induction. HA-FAK(Y397F) was transfected similarly into the dentate gyrus, and LTP was recorded $2 \mathrm{~d}$ later. The dentate gyrus tissue was dissected out $40 \mathrm{~min}$ after LTP induction for CREB determination. Results from Figure $4 B$ revealed that HA-FAK(Y397F) transfection produced a consistent decrease in fEPSP slope when compared with the pcDNA3 vector 
A
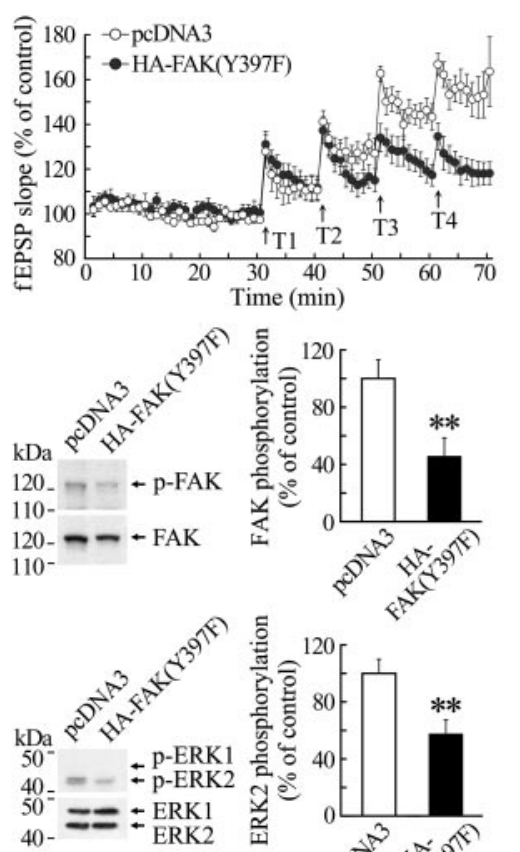

B

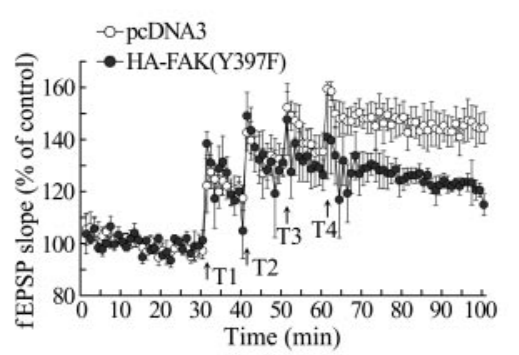

C

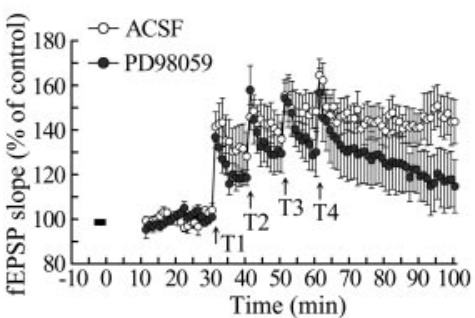

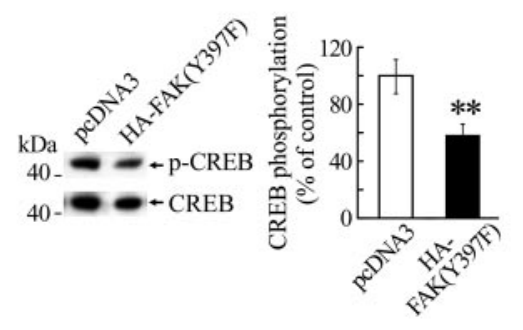
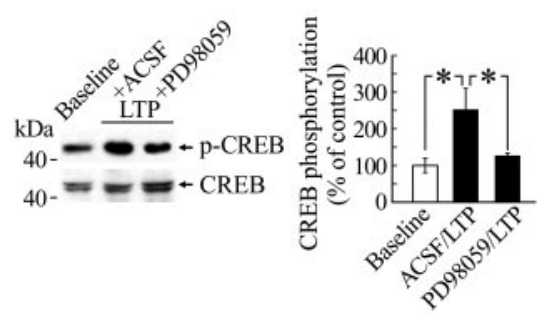

Figure 4. Transfection of HA-FAK(Y397F) impairs LTP induction and decreases FAK, ERK, and (REB phosphorylation. $A$, Transfection of HA-FAK(Y397F) markedly decreased the slope of fEPSP (top panel). Densitometry analyses revealed that HA-FAK(Y397F) transfection markedly decreased FAK phosphorylation (middle panel) and ERK2 phosphorylation (bottom panel; all $p<0.01$ ); $n=5-7$ for each group. $B$, Transfection of HA-FAK(Y397F) significantly decreased the slope of fEPSP and inhibited CREB phosphorylation (** $p<0.01) ; n=4-5$ for each group. C, PD98059 (75 $\mu$ m, $0.8 \mu l$ ) infused 10 min before LTP recording (horizontal black bar) decreased the slope of fEPSP at a late stage of LTP recording (91-100 min; $p<0.05$; top panel). Densitometry analysis revealed that tetanization increased CREB phosphorylation, and this effect was blocked by PD98059; $n=5$ for each group. Data are expressed as in Figure 1 ( $p<0.05$ and ** $p<0.01 ;$ one way AN0VA and Dunnett's $t$ test).

group [147 vs $126 \%$ of baseline, $n=5$ and $4\left(t_{(1,7)}=3.28 ; p<\right.$ $0.01)]$. Further analysis indicated that HA-FAK(Y397F) transfection also markedly decreased CREB phosphorylation $\left(t_{(1,7)}=\right.$ 3.18; $p<0.01)$ without significantly altering the CREB protein level (Fig. $4 B$ ). To clarify further whether CREB phosphorylation is a downstream event of MAPK/ERK activation, we infused a specific inhibitor of MAPK/ERK, PD98059, to the dentate gyrus 10 min before LTP recording. As shown in Figure 4C, PD98059 $(75 \mu \mathrm{M})$ impaired LTP at a relatively late stage of LTP recording $(91-100 \mathrm{~min})$ [ $114 \mathrm{vs} 146 \%$ of baseline, both $n=5\left(t_{(1,8)}=2.5\right.$; $p<0.05)]$. Further analysis revealed that there was a significant decrease in CREB phosphorylation in PD98059-treated rats 40 min after LTP induction $\left[\right.$ ACSF vs $\operatorname{PD98059~}\left(q_{(2,11)}=126 ; p<\right.$ 0.05); Fig. $4 C]$.

\section{Association of FAK $\left[\mathrm{pY}^{397}\right]$ with NR2A and NR2B is increased with tetanic stimulation}

The results above suggest that FAK plays an important role in the induction of LTP. Then how might FAK be involved in LTP induction? Previous studies have shown that the association of Fyn with FAK is increased with tetanic stimulation (Lauri et al., 2000). Further, Fyn is known to phosphorylate NMDARs in vivo (Kojima et al., 1998). It is possible that increased activation of FAK may enhance tyrosine phosphorylation of NMDARs. This experiment examined this hypothesis. As shown in Figure $5 A$, FAK $\left[\mathrm{pY}^{397}\right]$ is associated with NR1, NR2A, NR2B, and PI3kinase in hippocampal synaptosomal fraction under baseline stimulation. The association of FAK $\left[\mathrm{pY}^{397}\right]$ with NR2A and NR2B is increased with tetanic stimulation, but its association
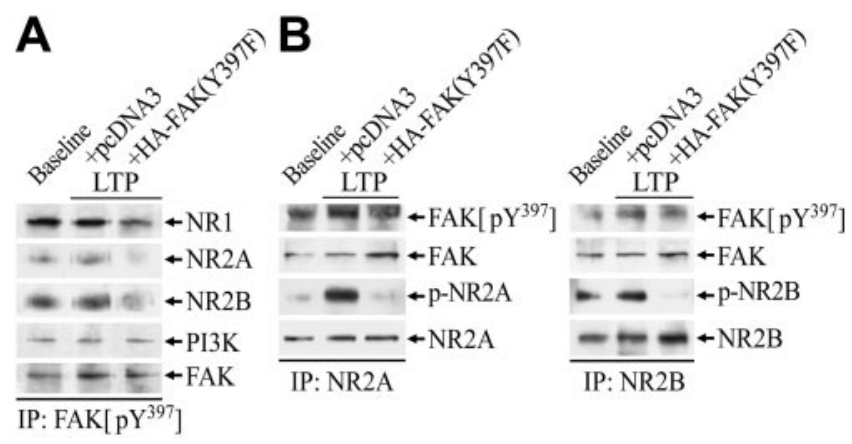

Figure 5. Tetanic stimulation enhances the association of FAK $\left[\mathrm{pY}^{397}\right]$ with NR2A and NR2B as well as the increased tyrosine phosphorylation of NR2A and NR2B. A, Precleared synaptosomal fraction $(100 \mu \mathrm{g})$ from dentate gyrus was immunoprecipitated with anti- FAK[pY $\left.{ }^{397}\right]$ antibody, followed by immunoblotting with anti-NR1, NR2A, NR2B, PI3-kinase, and FAK antibodies. Increased association with NR2A and NR2B was observed with tetanic stimulation. This association was decreased in the HA-FAK(Y397F)-transfected group. The association of FAK $\left[\mathrm{pY}^{397}\right.$ ] with NR1 and PI3-kinase remained similar. $B$, Immunoprecipitation was performed by using anti-NR2A and anti-NR2B antibodies, followed by immunoblotting with antiFAK $\left[\mathrm{pY}^{397}\right]$, FAK, phosphotyrosine, NR2A, and NR2B antibodies. Similarly, the association of FAK $\left[\mathrm{pY}^{397}\right.$ ] with NR2A and NR2B as well as the tyrosine phosphorylation of NR2A and NR2B was increased with tetanic stimulation. This increased association was prevented by HA-FAK(Y397F) transfection. The association of nonphosphorylated FAK with NR2A and NR2B was unchanged; $n=3-5$ for each group.

with NR1 and PI3-kinase remained unchanged. Further, transfection of the mutant construct HA-FAK(Y397F) prevented this increased association of FAK $\left[\mathrm{pY}^{397}\right]$ with NR2A and NR2B. To confirm further the obtained results, we performed immunopre- 
cipitation with NR2A and NR2B. As shown in Figure $5 B$, increased association of NR2A and NR2B with FAK[pY $\left.{ }^{397}\right]$ as well as increased tyrosine phosphorylation of NR2A and NR2B was observed with tetanic stimulation. This effect was blocked by HA-FAK(Y397F) transfection. Meanwhile, the FAK protein level remained similar in all groups.

\section{Discussion}

The present results have shown that both FAK phosphorylation and MAPK/ERK phosphorylation were increased 5 and $10 \mathrm{~min}$ after LTP induction in dentate gyrus neurons. Transfection of the HA-FAK(Y397F) construct impaired LTP induction, whereas transfection of the constitutively activated form $\operatorname{HA}-\mathrm{FAK}(\Delta 1-$ 100) reduced the threshold for LTP induction. FAK, MAPK/ERK, and CREB phosphorylation was decreased in HA-FAK(Y397F)transfected animals, and CREB phosphorylation was decreased with MAPK/ERK inhibition. These results suggest that FAK is required for LTP induction and that MAPK/ERK activation followed by CREB activation is the downstream event of FAK activation. Then how might FAK be involved in LTP induction? There are several possible explanations. First, FAK activation may enhance tyrosine phosphorylation of the NMDAR. This speculation is supported by the present finding that the association of phosphorylated FAK with NR2A and NR2B is increased with LTP induction, and this effect is blocked by HA-FAK (Y397F) transfection (Fig. 5). These results are consistent with the reports that phosphorylation of the NR2A and NR2B subunits is increased with LTP induction (Rosenblum et al., 1996; Rostas et al., 1996; Grosshans et al., 2001) and that upregulation of NMDAR current is mediated by tyrosine phosphorylation (Yu and Salter, 1999). Then how might FAK affect NMDAR? One possibility is that FAK phosphorylation may activate NMDAR directly and enhance NMDAR signaling that is required for LTP induction. However, this would require a direct measure of NMDAR current, and it awaits further investigation. Otherwise, FAK may modulate NMDAR activation via phosphorylation of other cytoplasmic proteins associated with the NMDAR, such as Fyn and Src (Yu et al., 1997). Further, the present results suggest that FAK probably does not interact with the NR1 subunit and PI3-kinase signaling pathway during LTP induction because the association of phosphorylated FAK with NR1 and PI3-kinase was not changed with tetanic stimulation. These results are consistent with the findings that NR2A and NR2B, but not NR1, subunits are phosphorylated on tyrosine residues (Lau and Huganir, 1995) and that PI3-kinase is required for the expression, but not the induction, of LTP in CA1 neurons (Sanna et al., 2002).

Second, FAK may modulate the activity of protein kinase C (PKC) that is required for LTP induction (Malinow et al., 1989; Wang and Feng, 1992). This explanation is supported by the finding that FAK promotes phospholipase C (PLC)- $\gamma 1$ activity (Zhang et al., 1999), and PLC is known to activate PKC via diacylglycerol. Third, FAK may be involved in cell adhesion molecule signaling, which plays an important role in synaptic plasticity and behavioral plasticity. For example, FAK is known as the primary mediator of integrin signaling (Hanks and Polte, 1997), whereas blockade of integrin function is known to impair LTP stabilization (Bahr et al., 1997) and memory consolidation in rats (Lee et al., 2000). Neural cell adhesion molecule (NCAM) is another member of the cell adhesion molecule family, and FAK is shown to mediate NCAM signaling involved in neurite outgrowth (Schmid et al., 1999). Because blockade of NCAM function is shown to impair LTP (Cremer et al., 1998) and spatial learning in rats (Arami et al., 1996), FAK also may mediate NCAM signaling involved in these functions. Alternatively, FAK may interact directly or indirectly with other cell adhesion molecules that also are involved in synaptic plasticity. For example, FAK mediates the signaling pathway of integrin-associated protein (Chung et al., 1997) that also is involved in LTP and memory process in rats and mice (Huang et al., 1998; Chang et al., 1999).

After demonstration of an increased association of FAK $\left[\mathrm{pY}^{397}\right]$ with NR2A and NR2B, we next asked the question whether FAK activation is a downstream event of NR2A and NR2B activation with LTP induction. Results revealed that blockade of NMDAR by D-APV did not prevent tetanization-induced increase in FAK, MAPK/ERK, and CREB phosphorylation. These results suggest that there may be multiple signaling pathways underlying LTP induction, but FAK, MAPK/ERK, and CREB are not downstream events of NMDAR activation. This suggestion is supported by the finding that the ERK cascade is required for NMDAR-independent LTP (Kanterewicz et al., 2000). Further, activation of the cholinergic system results in activation of the $\mathrm{fak}$ family (Lev et al., 1995), and coactivation of $\beta$-adrenergic and cholinergic receptors synergistically activates MAPK/ERK phosphorylation and enhances LTP induction (Watabe et el., 2000). Alternatively, FAK activation may occur before NMDAR activation because a fourfold increase in FAK phosphorylation was observed as soon as $5 \mathrm{~min}$ after LTP induction. In addition, the observed increase in FAK, MAPK/ERK, and CREB phosphorylation was specific to LTP because baseline stimulation and LFS did not produce the same biochemical changes. On the other hand, blockade of mGluR by MCPG did not block LTP induction. It also did not prevent tetanization-induced FAK phosphorylation. The lack of an effect of MCPG on LTP induction is congruent with the report that theta burst stimulation-induced LTP is insensitive to MCPG (Brown et al., 1994). In both cases strong tetanic stimulation was used. However, MCPG is shown to prevent weak stimulation-induced LTP. This is probably because, under weak stimulation, LTP induction requires the coactivation of NMDAR and mGluR, but under strong stimulation NMDAR activation is sufficient to induce LTP (Brown et al., 1994). These results together suggest that LTP-induced FAK phosphorylation is NMDAR- and mGluR-independent.

In the present study we have demonstrated that transfection of the HA-FAK(Y397F) construct impaired LTP, whereas transfection of the HA-FAK $(\Delta 1-100)$ construct reduced the threshold for LTP induction. What might be the mechanism underlying this reduced threshold? In analyzing the input-output relationship, we found that there is no significant difference in the amplitude of fEPSP between the control and HA-FAK $(\Delta 1-100)$ transfected group at any stimulus intensity that was examined. These results suggest that FAK does not change basal synaptic transmission of dentate gyrus neurons. FAK therefore may modulate the activity of other neurons to influence the threshold for LTP induction indirectly. This suggestion is supported by the finding that overexpression of $f y n$ reduces GABAergic inhibition in hippocampal neurons (Lu et al., 1999). On the other hand, although HA-FAK $(\Delta 1-100)$ transfection decreased the threshold for LTP induction, HA-FAK $(\Delta 1-100)$ itself did not induce longlasting potentiation (Fig. $2 \mathrm{~B}$ ). These results suggest that FAK is necessary, but not sufficient, to induce LTP. This result is different from the study showing that recombinant Src is sufficient to induce long-lasting potentiation and that this potentiation occludes LTP induction (Lu et al., 1998). This is probably attributable to a more direct interaction between Src and the NMDAR than with FAK (Ali and Salter, 2001; Cheung and Gurd, 2001).

The present results showed that MAPK/ERK phosphorylation 
also was increased markedly 5 and 10 min after LTP induction, a time course parallel with FAK activation. MAPK/ERK once was thought to contribute to a late stage of LTP; however, there is now more evidence showing that MAPK/ERK also is activated transiently with LTP induction, and it regulates short-term synaptic plasticity (English and Sweatt, 1996, 1997; Winder et al., 1999). Further, MAPK/ERK activation with LTP induction seems to be the downstream event of FAK activation because transfection of the FAK mutant construct impaired LTP induction and ERK2 phosphorylation. These results also suggest that MAPK may be involved in an early stage of LTP consolidation. Evidence supporting this notion comes from the observations that integrin antagonist blocks LTP stabilization (Bahr et al., 1997), and MAPK mediates integrin signaling (Chen and Guan, 1994). These results are also congruent with the concept that the MAPK/ ERK signaling cascade is critical for both neuronal plasticity and memory consolidation (Impey et al., 1999).

A number of studies have implicated that CREB is a nuclear target of MAPK/ERK activation and CREB is involved in various forms of long-term memory formation (Silva et al., 1998). In addition to MAPK/ERK phosphorylation 5-10 min after LTP induction, CREB phosphorylation was increased $40 \mathrm{~min}$ after LTP induction also. This result is consistent with the finding that CREB shows biphasic phosphorylation with LTP induction, with one peaked at $30 \mathrm{~min}$ and the other peaked at $2 \mathrm{hr}$ (Schulz et al., 1999). This result also shows a dissociation between MAPK/ERK phosphorylation and CREB phosphorylation and suggests that NMDA receptor activation followed by MAPK/ERK activation and CREB activation may not be an essential pathway required for all forms of LTP. This suggestion is supported by several findings. For example, Winder et al. (1999) have shown that MAPK/ERK is required for LTP elicited by brief low-frequency stimulation but is not required for LTP produced by certain highfrequency stimulation. In another study Watabe et al. (2000) have shown similarly that MAPK/ERK inhibition does not block LTP induced by certain high-frequency synaptic stimulation. These results together suggest that MAPK/ERK does not play an essential role in various forms of LTP induction. In addition, the CREB mutant mice (CREB $\alpha \Delta$ and CREBcomp) do not show deficits in hippocampal LTP induced by either low-frequency or high-frequency stimulation (Gass et al., 1998), suggesting that CREB may not be required for all forms of LTP either. Further, application of MAPK/ERK inhibitor blocked LTP induction and CREB phosphorylation, and transfection of HA-FAK(Y397F) construct decreased CREB phosphorylation. These results suggest that CREB is a downstream molecule of MAPK/ERK activation and FAK activation with LTP induction. It is also consistent with another study showing that the MAPK-CREB pathway is involved in the control of LTP-dependent gene expression (Davis et al., 2000). In the present study the effect of PD98059 on LTP and CREB phosphorylation is unlikely due to its effect on cell health and synaptic activity, because the concentration we used was $75 \mu \mathrm{M}$; however, at least $50 \mu \mathrm{M}$ PD98059 is needed to inhibit LTP, and $100 \mu \mathrm{M}$ was demonstrated not to affect basal synaptic transmission of hippocampal neurons (Coogan et al., 1999).

In summary, we have demonstrated that FAK plays an important role in LTP induction in dentate gyrus neurons in vivo and that MAPK/ERK and CREB are the downstream events of FAK activation. FAK may modulate LTP induction via enhanced tyrosine phosphorylation of NR2A and NR2B subunits. Whether FAK also is involved in learning and memory processes and other forms of synaptic plasticity requires further investigation.

\section{References}

Abdallah B, Hassan A, Benoist C, Goula D, Behr JP, Demeneix BA (1996) A powerful nonviral vector for in vivo gene transfer into the adult mammalian brain: polyethylenimine. Hum Gene Ther 7:1947-1954.

Abel T, Martin KC, Bartsch D, Kandel ER (1998) Memory suppressor genes: inhibitory constraints on the storage of long-term memory. Science 279:338-341.

Ali DW, Salter MW (2001) NMDA receptor regulation by Src kinase signaling in excitatory synaptic transmission and plasticity. Curr Opin Neurobiol 11:336-342.

Arami S, Jucker M, Schachner M, Welzl H (1996) The effect of continuous intraventricular infusion of L1 and NCAM antibodies on spatial learning in rats. Behav Brain Res 81:81-87.

Bahr BA, Staubli U, Xiao P, Chun D, Ji ZX, Esteban ET, Lynch G (1997) Arg-Gly-Asp-Ser-selective adhesion and the stabilization of long-term potentiation: pharmacological studies and the characterization of a candidate matrix receptor. J Neurosci 17:1320-1329.

Bashir ZI, Bortolotto ZA, Davies CH, Berreta N, Irving AJ, Seal AJ, Henley JM, Jane DE, Watkins JC, Collingridge GL (1993) The synaptic activation of glutamate metabotropic receptor is necessary for the induction of LTP in the hippocampus. Nature 363:347-350.

Boletta A, Benigni A, Lutz J, Remuzzi G, Soria MR, Monaco L (1995) Nonviral gene delivery to the rat kidney with polyethylenimine. Hum Gene Ther 8:1243-1251.

Brown RE, Rabe H, Reymann KG (1994) ( $R, S)$ - $\alpha$-methyl-4-carboxyphenylglycine (MCPG) does not block theta burst-induced long-term potentiation in area CA1 of rat hippocampal slices. Neurosci Lett 170:17-21.

Burgaya F, Menegon A, Menegoz M, Valtorta F, Girault JA (1995) Focal adhesion kinase in rat central nervous system. Eur J Neurosci 7:1810-1821.

Chang HP, Lindberg FP, Wang HL, Huang AM, Lee EHY (1999) Impaired memory retention and decreased long-term potentiation in integrinassociated protein-deficient mice. Learn Mem 6:448-457.

Chen HC, Guan JL (1994) Association of focal adhesion kinase with its potential substrate phosphatidylinositol 3-kinase. Proc Natl Acad Sci USA 91:10148-10152.

Cheung HH, Gurd JW (2001) Tyrosine phosphorylation of the N-methylD-aspartate receptor by exogenous and postsynaptic density-associated Src-family kinases. J Neurochem 78:524-534.

Chung J, Gao AG, Frazier WA (1997) Thrombospondin acts via integrinassociated protein to activate the platelet integrin $\alpha \operatorname{IIb} \beta 3$. J Biol Chem 272:14740-14746.

Collingridge GL, Bliss TV (1995) Memories of NMDA receptors and LTP. Trends Neurosci 8:54-56.

Coogan AN, O’Leary DM, O’Connor JJ (1999) P42/44 MAP kinase inhibitor PD98059 attenuates multiple forms of synaptic plasticity in rat dentate gyrus in vitro. J Neurophysiol 81:103-110.

Cremer H, Chazal G, Carleton A, Goridis C, Vincent JD, Lledo PM (1998) Long-term but not short-term plasticity at mossy fiber synapses is impaired in neural cell adhesion molecule-deficient mice. Proc Natl Acad Sci USA 95:13242-13247.

Davis S, Vanhoutte P, Pages C, Caboche J, Laroche S (2000) The MAPK/ ERK cascade targets both Elk-1 and cAMP response element-binding protein to control long-term potentiation-dependent gene expression in the dentate gyrus in vivo. J Neurosci 20:4563-4572.

English JD, Sweatt JD (1996) Activation of p42 mitogen-activated protein kinase in hippocampal long-term potentiation. J Biol Chem 271:2432924332.

English JD, Sweatt JD (1997) A requirement for the mitogen-activated protein kinase cascade in hippocampal long-term potentiation. J Biol Chem 272:19103-19106.

Furuta Y, Ilic D, Kanazawa S, Takeda N, Yamamoto T, Aizawa S (1995) Mesodermal defect in late phase of gastrulation by a targeted mutation of focal adhesion kinase, FAK. Oncogene 11:1989-1995.

Gass P, Wolfer DP, Balschun D, Rudolph D, Frey U, Lipp HP, Schutz G (1998) Deficits in memory tasks of mice with CREB mutations depend on gene dosage. Learn Mem 5:274-288.

Grant SGN, O’Dell TJ, Karl KA, Stein PL, Soriano P, Kandel ER (1992) Impaired long-term potentiation, spatial learning, and hippocampal development in fyn mutant mice. Science 258:1903-1910.

Grant SGN, Karl KA, Kiebler MA, Kandel ER (1995) Focal adhesion kinase in the brain: novel subcellular localization and specific regulation by Fyn tyrosine kinase in mutant mice. Genes Dev 9:1909-1921. 
Grosshans DR, Clayton DA, Coultrap SJ, Browning MD (2001) Proteomic analysis of NMDA receptor-adhesion protein signaling complexes. Nat Neurosci 5:27-33.

Hanks SK, Polte TR (1997) Signaling through focal adhesion kinase. BioEssays 19:137-145.

Huang AM, Wang HL, Tang YP, Lee EHY (1998) Expression of integrinassociated protein gene associated with memory formation in rats. J Neurosci 18:4305-4313.

Impey S, Obrietan K, Storm DR (1999) Making new connections: role of ERK/MAP kinase signaling in neuronal plasticity. Neuron 23:11-14.

Kanterewicz BI, Urban NN, McMahon DBT, Norman ED, Giffen LJ, Favata MF, Scherle PA, Trzaskos JM, Barrionuevo G, Klann E (2000) The extracellular signal-regulated kinase cascade is required for NMDA receptor-independent LTP in area CA1, but not area CA3, of the hippocampus. J Neurosci 20:3057-3066.

Koch CA, Anderson D, Moran MF, Ellis C, Pawson T (1991) SH2 and SH3 domains: elements that control interactions of cytoplasmic signaling proteins. Science 252:668-674.

Kojima N, Wang J, Mansuy IM, Grant SGN, Mayford M, Kandel ER (1997) Rescuing impairment of long-term potentiation in fyn-deficient mice by introducing Fyn transgene. Proc Natl Acad Sci USA 94:4761-4765.

Kojima N, Ishibashi H, Obata K, Kandel ER (1998) Higher seizure susceptibility and enhanced tyrosine phosphorylation of $N$-methyl-D-aspartate receptor subunit $2 \mathrm{~B}$ in $f y n$ transgenic mice. Learn Mem 5:429-445.

Lau LF, Huganir RL (1995) Differential tyrosine phosphorylation of N-methyl-D-aspartate receptor subunits. J Biol Chem 270:20036-20041.

Lauri SE, Taira T, Rauvala H (2000) High-frequency synaptic stimulation induces association of fyn and c-Src to distinct phosphorylated components. NeuroReport 11:997-1000.

Lee EH, Hsieh YP, Yang CL, Tsai KJ, Liu CH (2000) Induction of integrinassociated protein (IAP) mRNA expression during memory consolidation in rat hippocampus. Eur J Neurosci 12:1105-1112.

Lev S, Moreno H, Martinez R, Canoll P, Peles E, Musacchio JM, Plowman GD, Rudy B, Schlessinger J (1995) Protein tyrosine kinase PYK2 involved in $\mathrm{Ca}^{2+}$-induced regulation of ion channel and MAP kinase functions. Nature 376:737-745.

Lu YF, Kojima N, Tomizawa K, Moriwaki A, Matsushita M, Obata K, Matsui H (1999) Enhanced synaptic transmission and reduced threshold for LTP induction in fyn transgenic mice. Eur J Neurosci 11:75-82.

Lu YM, Roder JC, Davidow J, Salter MW (1998) Src activation in the induction of long-term potentiation in CA1 hippocampal neurons. Science 279:1363-1367.

Maguire C, Casey M, Kelly A, Mullany PM, Lynch MA (1999) Activation of tyrosine receptor kinase plays a role in expression of long-term potentiation in the rat dentate gyrus. Hippocampus 9:519-526.

Malinow R, Schulman H, Tsien RW (1989) Inhibition of postsynaptic PKC or CaMKII blocks induction but not expression of LTP. Science 245:862-866.

O’Dell TJ, Kandel ER, Grant SGN (1991) Long-term potentiation in the hippocampus is blocked by tyrosine kinase inhibitors. Nature 353:558-560.

Rosenblum K, Dudai Y, Richter-Levin G (1996) Modulation of protein ty- rosine phosphorylation in rat insular cortex after conditioned taste aversion training. Proc Natl Acad Sci USA 93:10457-10460.

Rosenblum K, Berman DE, Hazvi S, Lamprecht R, Dudai Y (1997) NMDA receptor and the tyrosine phosphorylation of its $2 \mathrm{~B}$ subunit in taste learning in the rat insular cortex. J Neurosci 17:5129-5135.

Rostas JA, Brent VA, Voss K, Errington ML, Bliss TV, Gurd JW (1996) Enhanced tyrosine phosphorylation of the $2 \mathrm{~B}$ subunit of the $\mathrm{N}$-methyl-Daspartate receptor in long-term potentiation. Proc Natl Acad Sci USA 93:10452-10456.

Sanna PP, Cammalleri M, Berton F, Simpson C, Lutjens R, Bloom FE, Francesconi W (2002) Phosphatidylinositol 3-kinase is required for the expression but not for the induction or the maintenance of long-term potentiation in the hippocampal CA1 region. J Neurosci 22:3359-3365.

Schlaepfer DD, Hunter T (1996) Evidence for in vivo phosphorylation of the Grb2 SH2-domain binding site on focal adhesion kinase by Src-family protein tyrosine kinases. Mol Cell Biol 16:5623-5633.

Schmid RS, Graff RD, Schaller MD, Chen S, Schachner M, Hemperly JJ, Maness PF (1999) NCAM stimulates the Ras-MAPK pathway and CREB phosphorylation in neuronal cells. J Neurobiol 38:542-558.

Schulz S, Siemer H, Krug M, Hollt V (1999) Direct evidence for biphasic camp responsive element-binding protein phosphorylation during longterm potentiation in the rat dentate gyrus in vivo. J Neurosci 19:5683-5692.

Siciliano JC, Toutant M, Derkinderen P, Sasaki T, Girault JA (1996) Differential regulation of proline-rich tyrosine kinase $2 /$ cell adhesion kinase $\beta$ $(\mathrm{PYK} 2 / \mathrm{CAK} \beta)$ and $\mathrm{pp} 125^{\text {(FAK) }}$ by glutamate and depolarization in rat hippocampus. J Biol Chem 271:28942-28946.

Silva AJ, Kogan JH, Frankland PW, Kida S (1998) CREB and memory. Annu Rev Neurosci 21:127-148.

Soderling TR, Derkach VA (2000) Postsynaptic protein phosphorylation and LTP. Trends Neurosci 23:75-80.

Wang HL, Wayner MJ, Chai CY, Lee EHY (1998) Corticotrophin-releasing factor produces a long-lasting enhancement of synaptic efficacy in the hippocampus. Eur J Neurosci 10:3428-3437.

Wang JH, Feng DP (1992) Postsynaptic protein kinase C is essential to induction and maintenance of long-term potentiation in the hippocampal CA1 region. Proc Natl Acad Sci USA 89:2576-2580.

Watabe AM, Zaki PA, O'Dell TJ (2000) Coactivation of $\beta$-adrenergic and cholinergic receptors enhances the induction of long-term potentiation and synergistically activates mitogen-activated protein kinase in the hippocampal CA1 region. J Neurosci 20:5924-5931.

Winder DG, Martin KC, Muzzio LA, Rohrer D, Chruscinski A, Kobilka B, Kandel ER (1999) ERK plays a regulatory role in induction of LTP by theta frequency stimulation and its modulation by $\beta$-adrenergic receptors. Neuron 24:715-726.

Yu XM, Salter MW (1999) Src, a molecular switch governing gain control of synaptic transmission mediated by $N$-methyl-D-aspartate receptors. Proc Natl Acad Sci USA 96:7697-7704.

Yu XM, Askalan R, Keil IIGJ, Salter MW (1997) NMDA channel regulation by channel-associated protein tyrosine kinase Src. Science 275:674-678.

Zhang X, Chattopadhyay A, Ji QS, Owen JD, Ruest PJ, Carpenter G, Hanks SK (1999) Focal adhesion kinase promotes phospholipase C- $\gamma 1$ activity. Proc Natl Acad Sci USA 96:9021-9026. 